\title{
An Observational Study to Assess the Prevalence of Hypertension and its Associated Disorders Among School Teachers in Dhaka City
}

\author{
*R Barua ${ }^{1}$, MA Noman², M Ismail ${ }^{3}$, MA Hossain $^{4}$
}

\begin{abstract}
Background: Urban school teachers are experiencing sedentary life style behaviors. This study was aimed to determine the prevalence of hypertension (HTN) and its relationship with associated disorders among school teachers of Dhaka city.

Methods: This cross sectional study was conducted between May-October of 2017. Total 323 school teachers in Dhaka city aged between 25-59 years were enrolled using cluster random sampling technique. Data on socio-demographic profile, personal and family history, co-morbidities and blood pressure were recorded. HTN was recorded according to predefined criteria.

Results: The prevalence of HTN among school teachers was $52.3 \%$. The mean \pm SD age was $46.5 \pm 7.8$ years. About $82 \%$ hypertensive teachers were within 41-59 years category. Majority participants were female $(65.6 \%)$ and female to male ratio was $1.9: 1$. Female teachers were more hypertensive compared to male (54.2\% vs $48.6 \%$ ). Majority teachers (52\%) were earning between 20,000 and 50,000 Bangladeshi Taka per month and about $61.6 \%$ had service duration $>15$ years. Male teachers with positive family history of diabetes mellitus (DM) were about one and half times likely to have DM as compared to negative family history $(\mathrm{p}=0.009, \mathrm{OR}=1.491,95 \%$ CI 1.138-1.954). Among hypertensive teachers, about $70 \%$ were overweight/obese and they were about 1.7 times likely to develop HTN $(p=0.024, \mathrm{OR}=1.696$ and 95\% CI 1.069-2.692) which was statistically significant.

Conclusions: HTN is more prevalent among urban school teachers and they are at greater risk to develop HTN. Building awareness by promoting health education, healthy life style and behaviors for prevention and control of HTN should be given importance among teachers.
\end{abstract}

Key Words: Hypertension, Prevalence, School teachers, Bangladesh

\section{Introduction}

Globally it has been experiencing epidemiological transition from communicable diseases to non communicable diseases (NCDs). Hypertension (HTN), is the major cause for NCDs such as cardiovascular diseases, heart failure, stroke, cancer etc and a leading risk factor for fetal and maternal deaths in pregnancy, dementia and renal failure. ${ }^{1}$ It

is a significant public health challenge and has a major impact on healthcare costs, contributing to around $10 \%$ of total healthcare spending globally. ${ }^{2}$ It imposes a serious economic burden on individuals, households, healthcare systems and the entire nation as a whole. ${ }^{3}$

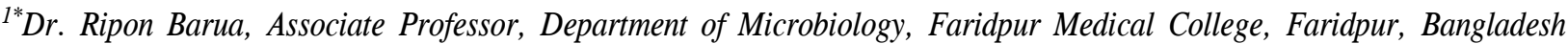
e-mail: riponbarua38cmc@gmail.com

${ }^{2}$ Dr. Mohammed Abu Noman, Department of Internal Medicine, Al Shamar Polyclinic, Barka, Oman

${ }^{3}$ Dr. Mohammad Ismail, Department of Community Medicine, Cox's Bazar Medical College, Cox's Bazar, Bangladesh

${ }^{4}$ Dr. Md. Amir Hossain, Department of Neurology, National Institute of Neurosciences and Hospital, Agargaon, Dhaka, Bangladesh
}

*Corresponding Author

Date of submission: 13.09.2018 Date of acceptance: 12.11 .2018

AKMMC J 2019; 10(1) : 70-76 
A recent analysis of worldwide data from different regions estimated that the total number of adults with hypertension in 2000 was 972 million: 333 million in countries with established market economies and 639 million in economically developing countries. This proportion will increase by $60 \%-1.56$ billion by $2025 .{ }^{4}$ The prevalence of HTN varies considerably by country: $20 \%$ in the USA and $25-50 \%$ in different regions in Europe. ${ }^{5}$ In India, it is $25 \%$ in urban area. ${ }^{6}$ According to the Bangladesh Non-communicable Disease Risk Factor Survey 2010, prevalence of HTN is $17.9 \%$ in general, $18.5 \%$ in men and $17.3 \%$ in women. ${ }^{7}$

HTN occurred in conjunction with a dramatic increase in the prevalence of overweight and obesity. ${ }^{8}$ HTN, diabetes mellitus (DM) and dyslipidaemia are important risk factors for atherosclerosis and its complications, including heart attacks and strokes. There is substantial overlap between HTN and other disorders, reflecting substantial overlap in their etiology and disease mechanisms. ${ }^{9}$

The teaching profession is highly stressful occupation due to enhanced psychosocial stress at the work place. The major sources of stress are colleagues, curriculum, parents, pupils, school authority, society, supervision/ teaching, teaching environment and income. ${ }^{10}$ School teachers in urban area are experiencing sedentary urban life style such as vehicle use for transport, environmental pollution, high calorie diet, lack of physical exercise etc. ${ }^{11}$ They play a vital role in learning process of the students that are very essential to build a prosperous nation and they must have a sound health status for this purpose.

This present study was aimed to determine the prevalence of HTN and its relationship with associated disorders among school teachers of Dhaka city, so that they can be aware regarding early detection, proper treatment and adequate control of HTN and its associated disorders.

\section{Materials \& Methods}

This was a cross sectional study. It was carried out in University of South Asia, Banani, Dhaka between May 2017 to October 2017. Mohammadpur area of
Dhaka city was the study area and the school teachers of that study area were the study population. All the teachers of selected schools aged between 25-59 years agreed to participate in this study were included. Teachers who had serious mental illness, pregnancy at any age group and not provided written informed consent were excluded.

For sample size calculation, the formula $\mathrm{N}=\mathrm{Z}^{2} \mathrm{pq} / \mathrm{d}^{2}$ was used. By considering the prevalence of HTN in urban area $30 \%,{ }^{12}$ acceptable limit of precision as $5 \%$ and $\mathrm{Z}$ value of 1.96 , the expected sample size came to be 323 . By adding $10 \%$ non response rate, sample size was calculated as 355 . The participants were enrolled using cluster random sampling technique. Mohammadpur area was divided into five zones. First School from each zone was selected randomly using the random number table and all the teachers were enrolled as defined criteria. 71 school teachers from each zone and total 355 teachers from five zones were intended to be enrolled. In case of inadequate sample from first institute, next institute(s) was selected randomly as described above. Due to some missing data and withdrawal from the study, total 323 participants were enrolled finally.

The study protocol was approved by the Institutional Ethics Committee of University of South Asia, Dhaka. All the teachers interviewed with the permission from the Principal or Head of schools. Before collecting the data, informed consent was taken from all study participants. They were assured that their given information should be kept confidential and they preserved the right to withdraw him/her from the study at any time without any threat or disadvantages. Any participants with HTN or other disorders referred to appropriate facilities.

To collect data, modified WHO STEPS protocol was followed. ${ }^{13}$ Demographic data including age, gender, education, occupation and income etc were collected in pretested and predesigned structured questionnaire for each participant. The interview included questions related to having medical disorders as well as personal and family history. Body weight, height and blood pressure (BP) were measured accordingly. 
Body weight was measured to the nearest $0.1 \mathrm{~kg}$ using a digital weight scale (Seca 803, Germany), which was calibrated weekly by using an object with known weight. Height was recorded to the nearest $0.1 \mathrm{~cm}$ in the standing position using a portable stadiometer. Body mass index (BMI) calculated using the formula, BMI = body weight $(\mathrm{kg}) /$ height $\left(\mathrm{m}^{2}\right)$. In this present study, the BMI of the study participants was classified according to WHO classification: a) underweight (BMI <18.5), b) normal weight (BMI 18.5-24.9), c) overweight (BMI 25-29.9) and obesity (BMI $\geqslant 30) .{ }^{13}$

BP measurements was followed a common protocol adapted from WHO Stepwise approach. ${ }^{13}$ Aneroid sphygmomanometer (ALPK2, Tanaka Sangyo Co. Ltd, Tokyo, Japan) with appropriately sized cuffs was used. BP was measured twice, five minute apart, with participants in a sitting position after five minutes of rest, in one visit. A third measurement was performed if the difference between the first two will be over $10 \mathrm{mmHg}$ for systolic or diastolic BP. The average of the second and the third BP measurements was used for analyses. In addition, participants were advised to avoid smoking, taking coffee/tea and performing any exercise for at least 30 minutes before measuring their BP. The Aneroid BP machine was calibrated against a mercury BP machine weekly. HTN was recorded as an average systolic blood pressure (SBP) $\geqslant 140 \mathrm{mmHg}$ and/or average diastolic blood pressure (DBP) $\geqslant 90 \mathrm{mmHg}$ and/or self-reported current treatment for HTN in the previous 2 weeks. ${ }^{14}$ Pre-HTN was defined by $\mathrm{SBP} \geqslant 120$ but $<140 \mathrm{mmHg}$ and/or $\mathrm{DBP} \geqslant 80$ but $<90 \mathrm{mmHg}$ and/or not taking anti-hypertensive medications and individuals with SBP $\leqslant 120$ or DBP $<80 \mathrm{mmHg}$ was considered as normotensive or non-HTN. ${ }^{14}$

All collected data were edited, checked and cleared manually, then interpreted by using computer based SPSS (Statistical Package for Social Science) software version 16.0 (Chicago, Illinois, USA) and Microsoft Excel 2007 version. Quantitative data were expressed as mean \pm standard deviation (SD) and dichotomous data represented as percentage. $\mathrm{p}<0.05$ was considered as statistically significant. Factors associated with HTN analyzed by
Chi-square test $\left(\chi^{2}\right)$. For analytical purposes, two groups (pre-HTN and non-HTN) were merged together to make the variable dichotomous (person with HTN versus non-HTN).

\section{Results}

This study was based on the observations of total 323 school teachers in Dhaka city. The mean \pm SD age of the participants was $46.5 \pm 7.8$ years and about $73 \%$ was aged 41-59 years of age group. Gender wise distribution showed that majority participants were female $(65.6 \%)$ and female to male ratio was 1.9:1 (Table 1). About $95 \%$ teachers were Muslim, 94\% married and $60 \%$ were highly educated as holding masters degree. Majority teachers $(52 \%)$ were in the middle income category earning between 20,000 and 50,000 Bangladeshi Taka per month and about $61.6 \%$ had service duration more than fifteen years (Table 1).

Out of total 323 teachers, $169(52.3 \%)$ were hypertensive and only $154(47.7 \%)$ were nonhypertensive. Among hypertensive teachers, female teachers were a little higher compared to male $(54.2 \%$ vs $48.6 \%)$ (Table 2). This study showed that $42 \%$ hypertensive teachers were within $41-50$ years category, followed by $40.2 \%$ and $17.2 \%$ among 51-59 and 31-40 years category respectively (Figure 1).

Male teachers with positive family history of DM were about one and half times likely to have DM as compared to negative family history $(\mathrm{p}=0.009$, $\mathrm{OR}=1.491,95 \%$ CI 1.138-1.954). This result was statistically significant. In the other hand, positive family history of DM among females and positive family history of HTN among both male and females were not likely to have DM and HTN as compared to negative family history $(\mathrm{p}=0.144$, OR $=1.193$, 95\% CI 0.948-1.501; $\mathrm{p}=0.844, \mathrm{OR}=0.974,95 \%$ CI $0.753-1.261$ and $\mathrm{p}=0.620, \mathrm{OR}=1.037,95 \%$ CI 0.897-1.199 respectively) (Table 3). 
Table 1: Sociodemographic characteristics of the study participants $(\mathrm{N}=323)$.

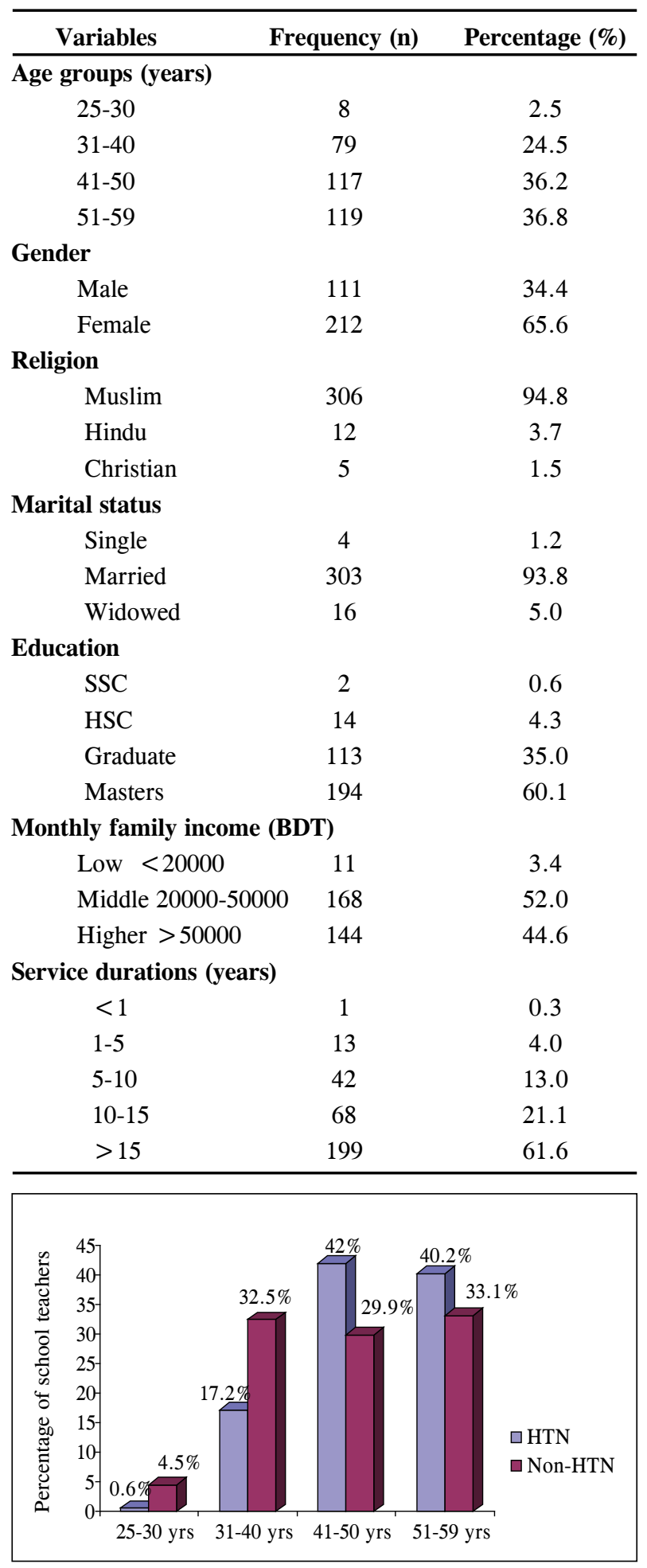

Figure 1: Distribution of hypertension $(\mathrm{HTN})$ as per age groups of the study participants.
Table 2: Prevalence of hypertension among school teachers in Dhaka city.

\begin{tabular}{|c|c|c|c|c|}
\hline Variable & $\begin{array}{c}\text { Male and } \\
\text { Female (323) } \\
\text { n (\%) }\end{array}$ & $\begin{array}{l}\text { Male } \\
(111) \\
\text { n (\%) }\end{array}$ & $\begin{array}{c}\text { Female } \\
(212) \\
\text { n }(\%)\end{array}$ & p-value \\
\hline \multicolumn{5}{|c|}{ Blood pressure measurement } \\
\hline Hypertension & $169(52.3)$ & $54(48.6)$ & $115(54.2)$ & \multirow{2}{*}{0.339} \\
\hline Non-Hypertension & $154(47.7)$ & $57(51.4)$ & $97(45.8)$ & \\
\hline
\end{tabular}

Table 3: Association between hypertension, diabetes mellitus and family history as per gender distribution among the study participants.

\begin{tabular}{lcccc}
\hline $\begin{array}{l}\text { Medical } \\
\text { condition }\end{array}$ & Gender & $\begin{array}{c}\text { Family history } \\
\text { positive (n) }\end{array}$ & $\begin{array}{c}\text { Odds ratio } \\
\text { (95\% CI) }\end{array}$ & p-value \\
\hline Hypertension & Male & 36 & $0.974(0.753-1.261)$ & 0.844 \\
& Female & 91 & $1.037(0.897-1.199)$ & 0.620 \\
Diabetes & Male & 27 & $1.491(1.138-1.954)$ & $0.009 *$ \\
mellitus & Female & 47 & $1.193(0.948-1.501)$ & 0.144 \\
\hline
\end{tabular}

N.B: * Indicates significant result. Analyzed by Chi-square test $\left(\chi^{2}\right)$.

Among hypertensive teachers, about $70 \%$ were overweight/obese and they were about 1.7 times likely to develop HTN ( $\mathrm{p}=0.024, \mathrm{OR}=1.696$ and $95 \%$ CI 1.0692.692). This result was statistically significant. In the other hand, about 33\% were diabetic and 29\% were dyslipidaemic among hypertensive teachers. But, there was no statistically significant difference observed between HTN, DM and dyslipidaemia $(\mathrm{p}=0.996$, $\mathrm{OR}=0.999,95 \%$ CI $0.628-1.589$ and $\mathrm{p}=0.229$, $\mathrm{OR}=1.357,95 \%$ CI 0.824-2.234 respectively) (Table 4).

Table 4: Hypertension (HTN) and its associated disorder among school teachers in Dhaka city.

\begin{tabular}{|c|c|c|c|c|}
\hline $\begin{array}{l}\text { Medical } \\
\text { condition }\end{array}$ & $\begin{array}{c}\text { HTN (151) } \\
\text { n (\%) }\end{array}$ & $\begin{array}{c}\text { Non-HTN } \\
(172) \\
\text { n (\%) }\end{array}$ & $\begin{array}{c}\text { Odds ratio } \\
\text { (95\% CI) }\end{array}$ & p-value \\
\hline \multicolumn{5}{|c|}{ Diabetes mellitus } \\
\hline Yes & $50(33.1)$ & $57(33.1)$ & $0.999(0.628-1.589)$ & 0.996 \\
\hline No & $101(66.9)$ & $115(66.9)$ & & \\
\hline \multicolumn{5}{|c|}{ Dyslipidaemia } \\
\hline Yes & $44(29.1)$ & $40(23.3)$ & $1.357(0.824-2.234)$ & 0.229 \\
\hline No & $107(70.9)$ & $132(76.7)$ & & \\
\hline \multicolumn{5}{|c|}{ Overweight/Obesity } \\
\hline Yes & $106(70.2)$ & $100(58.1)$ & $1.696(1.069-2.692)$ & $0.024 *$ \\
\hline No & $45(29.8)$ & $72(41.9)$ & & \\
\hline
\end{tabular}

N.B: *Indicates significant result. Analyzed by Chi-square test $\left(\chi^{2}\right)$. 


\section{Discussion}

This present study showed that among 323 school teachers of Dhaka city, about $52.3 \%$ had hypertension (HTN) (Table 2). The study finding was lower than that found in adults $(62.8 \%)$ in Nigeria $^{15}$ and higher compared with the studies conducted by Ibrahim NKR et al and Greiw AS et al which showed $25.2 \%$ and $15.1 \%$ prevalence of HTN among school teachers respectively. 16,17 This is also a bit higher than the previous study results estimated a prevalence rate of HTN among Bangladeshi adult ranging from $16-34 \% .{ }^{18}$ These differences may be due to urban residence, sedentary lifestyle of school teachers and lacking of large scale national survey of HTN among them.

Majority (42\%) hypertensive teachers were within 41-50 years category, followed by $40.2 \%$ and $17.2 \%$ among 51-59 and 31-40 years category respectively. The study relation to age with HTN signifies that as the age advances chances of becoming hypertensive are also increases (Figure 1). Similarly, the prevalence of HTN showed to be increased with increasing age $(\mathrm{p}=0.001)$ in the study by Ibrahim NKR et al in Jeddah. ${ }^{16}$ Zhao XL et al conducted a study in china and found positive and statistically significant association between advancing age and prevalence of HTN. 19

HTN was higher among female teachers (54.2\%) compared to male teachers $(48.6 \%)$ (Table 2). This study finding disagreed with that recorded in Nigeria, significant sex differences was established in blood pressure distribution ${ }^{15}$ and agreed with the study of Vyas et al and Girish B et al in India showed that female school teachers were hypertensive by $70.9 \%$ and $72.8 \%$ respectively. 20,21 Also different with that found in India by Sania et $\mathrm{al}$, the prevalence of HTN was higher (64\%) among male teachers. ${ }^{22}$

Majority teachers $(52 \%)$ were in the middle income category earning between 20,000 and 50,000 Bangladeshi Taka per month and about $61.6 \%$ had service duration more than fifteen years (Table 1). This study finding showed consistent results between income and HTN from many low and middle income countries. ${ }^{23}$ This finding indicates that in the context of the developing country having a higher income is not necessarily protective of health probably due to their lifestyle. These groups may use this income to provide more resources that may be used mostly for purchasing calorie-dense foods and in some instances it is a cause of sedentary lifestyles which are the underlying risk factors of HTN. About $95 \%$ teachers were Muslim, $94 \%$ married and $60 \%$ were highly educated as holding masters degree (Table 1).

Positive family history of DM among male teachers was about one and half times likely to have DM as compared to negative family history $(\mathrm{p}=0.009$, $\mathrm{OR}=1.491,95 \%$ CI 1.138-1.954). This result was statistically significant. But positive family history of DM among females and positive family history of HTN among both male and females were not likely to have DM and HTN as compared to negative family history $(\mathrm{p}=0.144, \mathrm{OR}=1.193,95 \% \mathrm{CI}$ $0.948-1.501 ; \mathrm{p}=0.844, \mathrm{OR}=0.974,95 \% \mathrm{CI}$ $0.753-1.261$ and $\mathrm{p}=0.620, \mathrm{OR}=1.037,95 \% \mathrm{CI}$ 0.897-1.199 respectively) (Table 3). This study finding disagree the result of the study conducted among adults in Turkey by Dogan et al showed a significant association between HTN and positive family history. ${ }^{24}$

This study showed that, among hypertensive teachers, about $70 \%$ were overweight/obese and they were about 1.7 times likely to develop HTN $(\mathrm{p}=0.024, \mathrm{OR}=1.696$ and $95 \%$ CI $1.069-2.692)$. This result was statistically significant. But, there was no statistically significant difference observed between HTN, DM and dyslipidaemia $(\mathrm{p}=0.996$, $\mathrm{OR}=0.999,95 \%$ CI $0.628-1.589$ and $\mathrm{p}=0.229$, $\mathrm{OR}=1.357,95 \%$ CI $0.824-2.234$ respectively) (Table 4). Ibrahim NKR et al in Jeddah had found high BMI was associated with HTN; the prevalence of HTN was $14.4 \%$ for normal weight teachers compared to $21.9 \%$ in overweight teachers and prevalence among obese individuals was $37.1 \%$ $(\mathrm{p}=0.001) .{ }^{16}$

\section{Conclusion}

It is concluded that HTN is more prevalent among urban school teachers and they are at greater risk to develop HTN due to their sedentary lifestyle. The 
increasing age has a significant role in the development of HTN. Overweight and obesity is directly related to development of HTN among urban school teachers.

\section{Recommendations}

School teachers should be regularly screened for early diagnosis, proper treatment and prevention of HTN. Awareness should be raised by promoting health education, healthy life style and behaviors. Longitudinal studies should be conducted among both urban and rural school teachers with a large sample size.

\section{Limitations}

Cross-sectional study design is the first limitation of this study as it limits the drawing of causal inferences. Secondly sample size was small. Finally, data were collected from schools of an urban area only not from any rural area resulting in difficulty in generalizing the study results.

\section{Acknowledgements}

Author would like to thank to all faculty members of the School of Public Health and Life Science, University of South Asia, Dhaka for their guidance and constructive criticism. We also acknowledge all the school teachers for their consent and active participation in this study.

\section{Conflict of interest: None}

\section{References}

1. Vasan RS, Beiser A, Seshadri S, et al. Residual lifetime risk for developing hypertension in middle-aged women and men: The Framingham Heart Study. JAMA 2002; 287(8): 1003-1010.

2. Lawes CM, Hoorn SV, Rodgers A. Global burden of blood-pressure-related disease, 2001. Lancet 2008; 371(9623): 1513-1518.

3. Karan A, Engelgau M, Mahal A. The householdlevel economic burden of heart disease in India. Trop Med \& Int Health 2014; 19(5): 581-591.
4. Kearney PM, Whelton M, Reynolds K, et al. Global burden of hypertension: analysis of worldwide data. Lancet 2005; 365(9455): 217 223.

5. TNS opinion and social health in European Union. Special Eurobarometer 272e/ Wave 66.2. September, 2007. Available at: http://ec.europa.eu/commfrontoffice /publicopinion/archives/ebs/ebs_272e_en.pdf. Accessed on $25^{\text {th }}$ May 2018.

6. Gupta R. Trends in hypertension epidemiology in India. J Hum Hypertens 2004; 18: 73-78.

7. World Health Organization. Bangladesh NCD risk factor survey 2010, WHO, Available at: h t t p : / / w w w. w h o b a n . or g/ Li n k Files/Publication_NCD_FACT_SHEET.pdf. Accessed on $28^{\text {th }}$ May 2018.

8. World health Organisation. Obesity: Preventing an launching the global epidemic-report of a who consultation, world health organ tech rep ser. 2000; 84: 1-253.

9. Cheung BM. The hypertension-diabetes continuum. J Cardiovasc Pharmacol 2010; 55: 333-339.

10. Olaitan L, Oyerinde O, Obiyemi $\mathrm{O}$, et al. Prevalence of job stress among primary school teachers in South-west, Nigeria. Afr $J$ Microbiology Research 2010; 4(5): 339-342.

11. Kovess-Masféty V, Sevilla-Dedieu C, RiosSeidel C, et al. Do teachers have more health problems? Results from a French crosssectional survey. BMC Public Health 2006; 6(101): 1-13.

12. Mohamed MR, Shafek M, El Damaty S, et al. Hypertension control indicators among rural population in Egypt. J Egypt Public Health Assoc 2000; 75(5-6): 391-401.

13. World Health Organization. WHO STEPS surveillance manual: the WHO STEP wise approach to chronic disease risk factor surveillance. 2005: WHO. 
14. Lenfant C, Chobanian AV, Jones DW, et al. Joint National Committee on the Prevention, Detection, Evaluation, and Treatment of High Blood Pressure. Seventh report of the Joint National Committee on the Prevention, Detection, Evaluation, and Treatment of High Blood Pressure (JNC 7) resetting the hypertension sails. Circulation 2003; 107(24): 2993-2994.

15. Uwah AF, Ndem JI, Emmanuel U. Prevalence of hypertension among adults attending Faithbased Centres in Abak Township, Akwa Ibom State, Nigeria. Merit Research J Med and Med Sci 2015; 3(7): 245-248.

16. Ibrahim NKR, Hijazi NA, Al- Bar AA. Prevalence and Determinants of Prehypertension and Hypertension among Preparatory and Secondary School Teachers in Jeddah. J Egypt Public Health Assoc. 2008; 83(3-4): 183-203.

17. Greiw ASH, Gad Z, Mandil A, et al. Risk Factors for Cardiovascular Diseases among School Teachers in Benghazi, Libya. Ibnosina $J$ Med BS. 2010; 2(4): 168-177.

18. Saquib $\mathrm{N}$, Saquib $\mathrm{J}$, Ahmed $\mathrm{T}$, et al. Cardiovascular diseases and type 2 diabetes in Bangladesh: a systematic review and metaanalysis of studies between 1995 and 2010 . BMC public health 1995; 12(1): 434. Available at: http:// www.biomedcentral.com/ 14712458/12/434. Accessed 20 th June 2018.
19. Zhao XL, Chen J, Cui YL, et al. Current status of primary hypertension in China an epidemiological study of 12 provinces 1 autonomous regions and 1 municipality. Zhonghua Yi Xue Za Zhi 2005; 85(40): 28302834.

20. Vyas PH, Bhate $\mathrm{K}$, Bawa $\mathrm{M}$, et al. A cross sectional study of epidemiological determinants correlated with prevalence of hypertension among municipal school teachers located in suburban area. Int J Com Med Public Health 2017; 4(2): 385-389.

21. Girish B, Sumanth MM. A study of hypertension \& its risk factors among primary school teachers of Tumkur, Karnataka. Indian J Forensic and Com Med 2017; 4(1): 53-57.

22. Sania S, Naila I. Lifestyle patterns and the prevalence of hypertension among the teachers of Kashmir University (Age 35 To 60 Yrs). Int J Home Sci 2017; 3(1): 150-154.

23. Ogah OS \& Rayner BL. "Recent advances in hypertension in sub-Saharan Africa," Heart 2013; 99(19): 1390-1397.

24. Dogan N, Toprak D, Demir S. Hypertension prevalence and risk factors among adult population in Afyonkarahisar region: a crosssectional research. Anadolu Kardiyol Derg 2012; 12: 47-52. 